\title{
Parameter Estimation of Lomax Distribution under Weighted Loss Function
}

Arun Kumar Rao, Himanshu Pandey*

Department of Mathematics \& Statistics, DDU Gorakhpur University, Gorakhpur, India

DOI: $10.36347 /$ sipms.2020.v07i07.005

| Received: 17.07.2020 | Accepted: 24.07.2020 | Published: 28.07.2020

*Corresponding author: Himanshu Pandey

Abstract

Review Article

Lomax distribution is considered. Bayesian method of estimation is employed in order to estimate the shape parameter of Lomax distribution by using quasi and gamma priors. In this paper, the Bayes estimators of the shape parameter have been obtained under squared error and weighted loss functions.

Keywords: Lomax distribution, Bayesian method, quasi and gamma priors squared error and weighted loss functions. Copyright @ 2020: This is an open-access article distributed under the terms of the Creative Commons Attribution license which permits unrestricted use, distribution, and reproduction in any medium for non-commercial use (NonCommercial, or CC-BY-NC) provided the original author and source are credited.

\section{INTRODUCTION}

Lomax distribution was introduced by Lomax [1]. The Lomax distribution is also known as Pareto distribution of second kind. It has been used in the analysis of income data and business failure data. It may describe the life time of a decreasing failure rate

$$
F(x ; \theta)=1-\left(1+\frac{x}{\lambda}\right)^{-\theta} ; x \geq 0, \theta>0 \text {. }
$$

Therefore, the probability density function of Lomax distribution is given by

$$
f(x ; \theta)=\frac{\theta}{\lambda}\left(1+\frac{x}{\lambda}\right)^{-(\theta+1)} \quad ; x \geq 0, \theta>0 .
$$

The joint density function or likelihood function of (2) is given by

$$
f(\underline{x} ; \theta)=\theta^{n} \lambda^{-n} e^{-(\theta+1) \sum_{i=1}^{n} \log \left(1+\frac{x_{i}}{\lambda}\right)}
$$

The log likelihood function is given by

$$
\log f(\underline{x} ; \theta)=n \log \theta-n \log \lambda-\theta \sum_{i=1}^{n} \log \left(1+\frac{x_{i}}{\lambda}\right)-\sum_{i=1}^{n} \log \left(1+\frac{x_{i}}{\lambda}\right)
$$

Differentiating (4) with respect to $\theta$ and equating to zero, we get the maximum likelihood estimator of $\theta$ as

$$
\hat{\theta}=\frac{n}{\sum_{i=1}^{n} \log \left(1+\frac{x_{i}}{\lambda}\right)}
$$




\section{Bayesian Method of Estimation}

In Bayesian analysis the fundamental problem are that of the choice of prior distribution $g(\theta)$ and a loss function $L(\hat{\theta}, \theta)$. The squared error loss function for the parameter $\theta$ are defined as:

$$
L(\hat{\theta}, \theta)=(\hat{\theta}-\theta)^{2}
$$

The Bayes estimator under the above loss function, say, $\hat{\theta}_{s}$ is the posterior mean, i.e,

$$
\hat{\theta}_{S}=E(\theta)
$$

This loss function is often used because it does not lead to extensive numerical computations but several authors Zellner [3], Basu and Ebrahimi [4], Norstrom [5] have recognized that the inappropriateness of using symmetric loss function. Ahamad et al., [6] introduced weighted loss function which is given as:

$$
L(\hat{\theta}, \theta)=\frac{(\hat{\theta}-\theta)^{2}}{\theta}
$$

The Bayes estimator under weighted loss function is denoted by $\hat{\theta}_{W}$ and is obtained as

$$
\hat{\theta}_{W}=\left[E\left(\frac{1}{\theta}\right)\right]^{-1}
$$

Let us consider two prior distributions of $\theta$ to obtain the Bayes estimators.

(i) Quasi-prior: For the situation where the experimenter has no prior information about the parameter $\theta$, one may use the quasi density as given by

$$
g_{1}(\theta)=\frac{1}{\theta^{d}} ; \theta>0, d \geq 0,
$$

Where $d=0$ leads to a diffuse prior and $d=1$, a non-informative prior.

(ii) Gamma prior: The most widely used prior distribution of $\theta$ is the gamma distribution with parameters $\alpha$ and $\beta(>0)$ with probability density function given by

$$
g_{2}(\theta)=\frac{\beta^{\alpha}}{\Gamma(\alpha)} \theta^{\alpha-1} e^{-\beta \theta} ; \theta>0
$$

\section{Bayes Estimators under $g_{1}(\theta)$}

The posterior density of $\theta$ under $g_{1}(\theta)$, on using (3), is given by

$$
f(\theta / \underline{x})=\frac{\theta^{n} \lambda^{-n} e^{-(\theta+1) \sum_{i=1}^{n} \log \left(1+\frac{x_{i}}{\lambda}\right)} \theta^{-d}}{\int_{0}^{\infty} \theta^{n} \lambda^{-n} e^{-(\theta+1) \sum_{i=1}^{n} \log \left(1+\frac{x_{i}}{\lambda}\right)} \theta^{-d} d \theta}
$$




$$
\begin{aligned}
& =\frac{\theta^{n-d} e^{-\theta \sum_{i=1}^{n} \log \left(1+\frac{x_{i}}{\lambda}\right)}}{\int_{0}^{\infty} \theta^{n-d} e^{-\theta \sum_{i=1}^{n} \log \left(1+\frac{x_{i}}{\lambda}\right)} d \theta} \\
& =\frac{\left(\sum_{i=1}^{n} \log \left(1+\frac{x_{i}}{\lambda}\right)\right)^{n-d+1}}{\Gamma(n-d+1)} \theta^{n-d} e^{-\theta \sum_{i=1}^{n} \log \left(1+\frac{x_{i}}{\lambda}\right)}
\end{aligned}
$$

Theorem 1. Assuming the squared error loss function, the Bayes estimate of the shape parameter $\theta$, is of the form

$$
\hat{\theta}_{S}=\frac{n-d+1}{\sum_{i=1}^{n} \log \left(1+\frac{x_{i}}{\lambda}\right)}
$$

Proof. From equation (7), on using (12),

$$
\begin{aligned}
& \hat{\theta}_{S}=E(\theta)=\int \theta f(\theta / \underline{x}) d \theta \\
& =\frac{\left(\sum_{i=1}^{n} \log \left(1+\frac{x_{i}}{\lambda}\right)\right)^{n-d+1}}{\Gamma(n-d+1)} \int_{0}^{\infty} \theta^{n-d+1} e^{-\theta \sum_{i=1}^{n} \log \left(1+\frac{x_{i}}{\lambda}\right)} d \theta \\
& =\frac{\left(\sum_{i=1}^{n} \log \left(1+\frac{x_{i}}{\lambda}\right)\right)^{n-d+1}}{\Gamma(n-d+1)} \frac{\Gamma(n-d+2)}{\left(\sum_{i=1}^{n} \log \left(1+\frac{x_{i}}{\lambda}\right)\right)^{n-d+2}} \\
& \text { or, } \hat{\theta}_{S}=\frac{(n-d+1)}{\sum_{i=1}^{n} \log \left(1+\frac{x_{i}}{\lambda}\right)} .
\end{aligned}
$$

Theorem 2. Assuming the weighted loss function, the Bayes estimate of the shape parameter $\theta$, is of the form

$$
\hat{\theta}_{W}=\frac{n-d}{\sum_{i=1}^{n} \log \left(1+\frac{x_{i}}{\lambda}\right)} \text {. }
$$

Proof. From equation (9), on using (12),

$$
\begin{aligned}
& \hat{\theta}_{W}=\left[E\left(\frac{1}{\theta}\right)\right]^{-1}=\left[\int \frac{1}{\theta} f(\theta / \underline{x}) d \theta\right]^{-1} \\
& =\left[\frac{\left(\sum_{i=1}^{n} \log \left(1+\frac{x_{i}}{\lambda}\right)\right)^{n-d+1}}{\Gamma(n-d+1)} \int_{0}^{\infty} \theta^{n-d-1} e^{-\theta \sum_{i=1}^{n} \log \left(1+\frac{x_{i}}{\lambda}\right)} d \theta\right]^{-1}
\end{aligned}
$$




$$
\begin{aligned}
& =\left[\frac{\left(\sum_{i=1}^{n} \log \left(1+\frac{x_{i}}{\lambda}\right)\right)^{n-d+1}}{\Gamma(n-d+1)} \frac{\Gamma(n-d)}{\left(\sum_{i=1}^{n} \log \left(1+\frac{x_{i}}{\lambda}\right)\right)^{n-d}}\right]^{-1} \\
& =\left[\frac{\sum_{i=1}^{n} \log \left(1+\frac{x_{i}}{\lambda}\right)}{n-d}\right]^{-1}
\end{aligned}
$$

or, $\hat{\theta}_{W}=\frac{n-d}{\sum_{i=1}^{n} \log \left(1+\frac{x_{i}}{\lambda}\right)}$.

\section{Bayes Estimators under $g_{2}(\theta)$}

Under $g_{2}(\theta)$, the posterior density of $\theta$, using equation (3), is obtained as

$$
\begin{aligned}
& f(\theta / \underline{x})=\frac{\theta^{n} \lambda^{-n} e^{-(\theta+1) \sum_{i=1}^{n} \log \left(1+\frac{x_{i}}{\lambda}\right)} \frac{\beta^{\alpha}}{\Gamma(\alpha)} \theta^{\alpha-1} e^{-\beta \theta}}{\int_{0}^{\infty} \theta^{n} \lambda^{-n} e^{-(\theta+1) \sum_{i=1}^{n} \log \left(1+\frac{x_{i}}{\lambda}\right)} \frac{\beta^{\alpha}}{\Gamma(\alpha)} \theta^{\alpha-1} e^{-\beta \theta} d \theta} \\
& =\frac{\theta^{n+\alpha-1} e^{-\left(\beta+\sum_{i=1}^{n} \log \left(1+\frac{x_{i}}{\lambda}\right)\right) \theta}}{\int_{0}^{\infty} \theta^{n+\alpha-1} e^{-\left(\beta+\sum_{i=1}^{n} \log \left(1+\frac{x_{i}}{\lambda}\right)\right) \theta} d \theta} \\
& =\frac{\theta^{n+\alpha-1} e^{-\left(\beta+\sum_{i=1}^{n} \log \left(1+\frac{x_{i}}{\lambda}\right)\right) \theta}}{\Gamma(n+\alpha) /\left(\beta+\sum_{i=1}^{n} \log \left(1+\frac{x_{i}}{\lambda}\right)\right)^{n+\alpha}} \\
& =\frac{\left(\beta+\sum_{i=1}^{n} \log \left(1+\frac{x_{i}}{\lambda}\right)\right)^{n+\alpha}}{\Gamma(n+\alpha)} \theta^{n+\alpha-1} e^{-\left(\beta+\sum_{i=1}^{n} \log \left(1+\frac{x_{i}}{\lambda}\right)\right) \theta} \ldots
\end{aligned}
$$

Theorem 3. Assuming the squared error loss function, the Bayes estimate of the shape parameter $\theta$, is of the form

$$
\hat{\theta}_{S}=\frac{n+\alpha}{\beta+\sum_{i=1}^{n} \log \left(1+\frac{x_{i}}{\lambda}\right)}
$$

Proof. From equation (7), on using (15),

$$
\hat{\theta}_{S}=E(\theta)=\int \theta f(\theta / \underline{x}) d \theta
$$




$$
\begin{aligned}
& =\frac{\left(\beta+\sum_{i=1}^{n} \log \left(1+\frac{x_{i}}{\lambda}\right)\right)^{n+\alpha}}{\Gamma(n+\alpha)} \int_{0}^{\infty} \theta^{n+\alpha} e^{-\left(\beta+\sum_{i=1}^{n} \log \left(1+\frac{x_{i}}{\lambda}\right)\right) \theta} d \theta \\
& =\frac{\left(\beta+\sum_{i=1}^{n} \log \left(1+\frac{x_{i}}{\lambda}\right)\right)^{n+\alpha}}{\Gamma(n+\alpha)} \frac{\Gamma(n+\alpha+1)}{\left(\beta+\sum_{i=1}^{n} \log \left(1+\frac{x_{i}}{\lambda}\right)\right)^{n+\alpha+1}}
\end{aligned}
$$

or, $\quad \hat{\theta}_{S}=\frac{n+\alpha}{\beta+\sum_{i=1}^{n} \log \left(1+\frac{x_{i}}{\lambda}\right)}$

Theorem 4. Assuming the weighted loss function, the Bayes estimate of the shape parameter $\theta$, is of the form

$$
\hat{\theta}_{W}=\frac{n+\alpha-1}{\beta+\sum_{i=1}^{n} \log \left(1+\frac{x_{i}}{\lambda}\right)}
$$

Proof. From equation (9), on using (15),

$$
\begin{aligned}
& \hat{\theta}_{W}=\left[E\left(\frac{1}{\theta}\right)\right]^{-1}=\left[\int \frac{1}{\theta} f(\theta / \underline{x}) d \theta\right]^{-1} \\
& =\left[\frac{\left(\beta+\sum_{i=1}^{n} \log \left(1+\frac{x_{i}}{\lambda}\right)\right)^{n+\alpha}}{\Gamma(n+\alpha)} \int_{0}^{\infty} \theta^{n+\alpha-2} e^{-\left(\beta+\sum_{i=1}^{n} \log \left(1+\frac{x_{i}}{\lambda}\right)\right)_{\theta}} d \theta\right]^{-1} \\
& =\left[\frac{\left(\beta+\sum_{i=1}^{n} \log \left(1+\frac{x_{i}}{\lambda}\right)\right)^{n+\alpha}}{\Gamma(n+\alpha)} \frac{\Gamma(n+\alpha-1)}{\left(\beta+\sum_{i=1}^{n} \log \left(1+\frac{x_{i}}{\lambda}\right)\right)^{n+\alpha-1}}\right]^{-1} \\
& =\left[\frac{\left.\beta+\sum_{i=1}^{n} \log \left(1+\frac{x_{i}}{\lambda}\right)\right]^{-1}}{n+\alpha-1}\right]^{-1} \\
& \text { or, } \hat{\theta}_{W}=\frac{[+\alpha-1}{\beta+\sum_{i=1}^{n} \log \left(1+\frac{x_{i}}{\lambda}\right)} .
\end{aligned}
$$

\section{CONCLUSION}

In this paper, we have obtained a number of estimators of parameter of Lomax distribution. In equation (13) and (14) we have obtained the Bayes estimators under squared error and weighted loss functions using quasi prior. In equation (16) and (17) we have obtained the Bayes estimators under squared error and weighted loss functions using gamma prior. In the above equation, it is clear that the Bayes estimators depend upon the parameters of the prior distribution.

\section{REFERENCES}

1. Lomax, K. S. (1954). Business failures: Another example of the analysis of failure data. Journal of 
the American Statistical Association, 49(268), 847-852.

2. Ahmad, A., Ahmad, S. P., \& Ahmed, A. (2016) Bayesian Analysis of Lomax Distribution under Asymmetric Loss Functions. Journal of Statistics Applications and Probability Letters, 3(1), 35-44.

3. Zellner, A. (1986). Bayesian estimation and prediction using asymmetric loss functions. Journal of the American Statistical Association, 81(394), 446-451.

4. Basu, A. P., \& Ebrahimi, N. (1991). Bayesian approach to life testing and reliability estimation using asymmetric loss function. Journal of statistical planning and inference, 29(1-2), 21-31.

5. Norstrom, J. G. (1996). The use of precautionary loss functions in risk analysis. IEEE Transactions on reliability, 45(3), 400-403.

6. Ahmad, S. P., Ahmad, A., \& Ahmed, A. (2016). Bayesian Estimation for the Class of Life-Time Distributions Under Different Loss Functions. Statistics and Applications, 14(1-2), 7591. 